Forum 2020 · 35:300-303

https://doi.org/10.1007/s12312-020-00804-0

Online publiziert: 3 . Juli 2020

(c) Springer Medizin Verlag GmbH, ein Teil von Springer Nature 2020

Meine Krankheit, meine DNA, meine Therapie. Die Medizin wird sich in den kommenden Jahren weiter personalisieren. Die Aufbruchstimmung erfasst nicht nur Wissenschaftler und Onkologen, sondern auch ITund Datenspezialisten. Denn für eine individualisierte Diagnostik und Therapie sind mehrere Voraussetzungen zu erfüllen: Neben einem vertieften Verständnis der biologischen Grundlagen und dem Instrumentarium, diese zu entschlüsseln, muss der umfassende Zugriff auf Daten sowie deren Weiterverarbeitung gewährleistet sein. Darüber hinaus bedarf es der notwendigen technologischen und personellen Ressourcen, um die richtigen therapeutischen Schlüsse aus der vorhandenen Datenflut zu ziehen.

Nach Jahren der Trägheit hat die Digitalisierung nun auch die Gesundheitsbranche in Deutschland erfasst, und das mit einer bislang unbekannten Dynamik. Gewissermaßen über Nacht ist „digital“ zum Synonym von „innovativ“ geworden. Egal, ob Pflegenotstand, Hygieneprobleme oder eine bessere, weil präzisere Behandlung bei Tumorerkrankungen - die Digitalisierung als omnipotentes, Heil bringendes Versprechen ist allgegenwärtig.

Prof. Dr. med.
Jörg F. Debatin
Bundesministerium für
Gesundheit, Berlin

Jörg F. Debatin · Claudia Dirks

hih - health innovation hub, Bundesministerium für Gesundheit, Berlin, Deutschland

\title{
Die digitale Transformation der Medizin
}

Eine Näherung an die Leiden des Menschen auf molekularer Ebene ist zweifelsfrei die nächste große Revolution in der Medizin. Wurden Tumorerkrankungen bislang organ- bzw. zellspezifisch diagnostiziert und therapiert, zielt die Medizin der Zukunft nun auf eine Ebene tiefer. Technisch und wirtschaftlich möglich geworden, ist die Entschlüsselung genetischer Grundlagen von Wirt- und Tumorzellen mithilfe der Sequenziermethoden der nächsten Generation („next generation sequencing"). Die Analyse der gewonnenen Datenfluten gelingt nur mit Unterstützung spezialisierter Tools. So sind v.a. in der Onkologie stratifizierte bzw. individualisierte Ansätze schon am weitesten vorangeschritten. Die digitale Dynamik beschränkt sich aber nicht nur auf die Onkologie, sondern betrifft alle Bereiche der Medizin.

Angetrieben werden diese fundamentalen Veränderungen in der Gesundheitsversorgung von drei zentralen Entwicklungen:

1. besseres Verständnis der biologischen Lebensgrundlagen,

2. technologische Quantensprünge bei Computing und Datenspeicherung, gepaart mit dem ubiquitären Zugang zu Daten über Smartphones, sowie

3. deutlich gestiegenes Souveränitätsbedürfnis der Patienten auf der Basis zunehmender Transparenz und digitaler Kommunikation.

\section{Treiber der digitalen Transformation}

Biologische Lebensgrundlagen. Zwischen zwei Menschen sind 99,5\% des genetischen Codes (DNA) identisch. Obgleich schwer vorstellbar, gilt das sogar für die Paarung „Donald Trump und Kim Jong-Un“; oder vielleicht ist es gerade bei diesen beiden gut vorstellbar. Diese Erkenntnis stellt die Grundlage für Zulassungen von Medikamenten und Behandlungsleitlinien dar. Die Medizin ist standardisiert; optimale Resultate werden nur dann erreicht, wenn alle in gleicher Weise behandelt werden - so die bisherige Logik.

Da die DNA immer schneller und kostengünstiger entschlüsselt werden kann, rücken die differenzierenden $0,5 \%$ des genetischen Codes in den Fokus. Diese $0,5 \%$ unterschiedliche Wirt-DNA umfasst bis zu 3 Mio. Basenpaare in jeder Zelle. In Anbetracht von 3 Mio. Differenzierungskriterien erscheint es allerdings recht unwahrscheinlich, dass zwei Menschen auf eine komplexe medikamentöse Therapie in vollkommen identischer Weise reagieren. Hinzu kommen Mutationen der Zellen, in denen sich der genetische Code gänzlich verändert und sich in Tumorzellen entwickeln. Darüber hinaus wird der Organismus durch Umwelteinflüsse geprägt, die zu erheblichen Unterschieden in der Epigenetik führen.

Nachdem die biologischen Grundlagen menschlichen Lebens mit zunehmend überschaubarem zeitlichen und wirtschaftlichen Aufwand entschlüsselt werden können, nehmen die gemessenen Unterschiede auch für die medizinische Diagnostik und Therapie an Relevanz deutlich zu. Inzwischen ist klar: Die Einzigartigkeit des menschlichen Organismus sowie der dazugehörigen Erkrankungen bedürfen individualisierter Therapien. Diese Erkenntnis ist die Basis der „personalisierten Medizin“, die, wie eingangs hervorgehoben, gerade in der modernen Onkologie bereits heute eine herausragende Rolle spielt. 
Die vielen Millionen differenzierender Einflussgrößen überfordern in ihrer Anzahl und Komplexität das menschliche Gehirn und damit auch jeden Arzt. Um für jeden Einzelnen das persönliche Therapieoptimum zu identifizieren, bedarf es zunehmend der umfassenden Unterstützung durch Computing und $\mathrm{Da}$ tenspeicherung. Eine personalisierte, auf den individuellen Menschen mit seinen Erkrankungen abgestimmte Therapie ist ohne digitale Unterstützung nicht realisierbar.

\section{Quantensprünge in der Digitaltechno-} logie. Das Cloud-Computing hat eine neue Grundlage für die Nutzung digitaler Technologien geschaffen. Für Anwender auf der ganzen Welt sind eine beinahe unbegrenzte Rechenleistung und unbegrenzte Speicherkapazitäten verfügbar geworden. Hinzu kommt der fast ubiquitäre Zugang zu Daten in der Cloud über mobile Endgeräte. Damit ist jeder Einzelne in der Lage, diese Technologien für sich und andere zu nutzen. Für die Dokumentation und Speicherung medizinischer Daten ermöglicht diese technologische Innovation einen fundamentalen Paradigmenwechsel: Hatten medizinische Daten bislang immer einen direkten Bezug zu ihrem Entstehungsort, an dem sie i. d. R. auch gespeichert wurden, können sie nun zentral, unabhängig von ihrem Entstehungsort, patientenspezifisch gespeichert und verarbeitet werden. Nicht mehr der Ort der Datenakquisition ist ausschlaggebend, sondern der jeweilige Patient, von dem die Daten stammen. Über mobile Endgeräte ist zudem jedes (berechtigte) Individuum in der Lage, diese Daten abzurufen, zu analysieren und weiterzuverarbeiten.

Die Cloud-basierte Explosion der Rechenleistung mit schier unbegrenzten Datenspeicherkapazitäten, gepaart mit ubiquitärem, dezentralem und mobilem Datenzugang ist der technologische Treiber der anstehenden digitalen Revolution in der Medizin.

\section{Gestiegene Patientensouveränität.}

Angst und Sorge vor Krankheit und Tod haben das Thema "Gesundheit“ über Jahrhunderte mystifiziert. Patienten „begaben sich in Behandlung“, oftmals im blinden Vertrauen auf ihre Ärzte. Durch den Einsatz einer eigenen Sprache trugen die Mediziner nachhaltig zur Mystifizierung der eigenen Heilkunst bei. Transparenz war von den Patienten nicht gefordert und von den Ärzten nicht gewollt. Der „mündige Patient“ ist ein eher neues Phänomen, dass sich allerdings rasch weiterentwickelt. Diesbezüglich hat die Coronavirus-Pandemie wahrscheinlich die größten Veränderungen hervorgerufen. Das Informationsmonopol der Ärzte wird durch Apps, Bots und andere Tools immer mehr infrage gestellt. Patienten sind zunehmend besser informiert. Auf dieser Basis beund hinterfragen sie ihre behandelnden Ärzte und Therapeuten.

Gleichzeitig erleben die Menschen die Vorzüge der Digitalisierung im Alltag. Direkte Kommunikation mit ihrem Arzt, ohne die eigene Wohnung zu verlassen, die elektronische Arbeitsunfähigkeitsbescheinigung, das digital erneuerbare Rezept - auf diese, in der Pandemie erlebten digitalen Versorgungsangebote wollen viele auch nach der Krise nicht verzichten.

Die digitale Terminvergabe beim Arzt wird ebenso zum Standard wie Onlinebanking oder andere Alltagshandlungen. Die Menschen werden im Umgang mit dem Gut „Gesundheit“ souveräner und anspruchsvoller. Um im Markt zu überleben, werden sich die medizinischen Leistungserbringer auf noch mehr Teamarbeit und Transparenz einstellen müssen - und das zunehmend online.

Diese drei Treiber bilden ein Team und werden die Medizin in den kommenden Jahren dank digitaler Optionen grundlegend verändern. Doch wie andere Technologien, derer sich die Medizin bedient, ist auch die Digitalisierung lediglich das Mittel zum Zweck und die technische Grundlage für eine bessere Gesundheitsversorgung der Menschen durch ein Mehr an Qualität und Effizienz in der Medizin. Ohne das enorme Potenzial der personalisierten Medizin bezüglich einer qualitativ besseren Versorgung und das Verlangen der Menschen nach Transparenz, Effizienz und Bequemlichkeit wären digitale Technologien reiner Selbstzweck. Der Nutzen für die Menschen prägt den von Weidenfeld und Nieder-Rümelin beschriebenen „digitalen Humanismus", bei dem weiterhin der Mensch und nicht die Technologie im Mittelpunkt steht [1].

Doch nur mit dem breiten Einsatz digitaler Technologien kann den berechtigten Ansprüchen informierter Patienten auf Sicherheit und Transparenz in Kombination mit einer für den Einzelnen optimierten, also personalisierten Medizin, Rechnung getragen werden.

\section{Die elektronische Patientenakte als Grundlage für Innovationen}

Die meisten für eine zielgenaue Therapie notwendigen relevanten Daten existieren bereits, sie sind allerdings nicht miteinander verbunden. Damit fehlt jeglicher Überblick. Um sicherzustellen, dass sich die Medizin im Sinne der Menschen wirklich verbessert, muss dieser Mangel dringend behoben werden. Die bestehenden Datensilos, die i. d. R. bei den Leistungserbringern untergebracht sind, müssen strukturiert zusammengezogen werden. Diese zentrale Aufgabe soll die elektronische Patientenakte (ePA) übernehmen, deren Einführung zum 1. Januar 2021 avisiert ist.

Die ePA stellt die Grundlage für einen vielfältigen weiteren Nutzen dar. Ohne sie und ihre erfolgreiche Implementierung werden die Potenziale einer digitalen Medizin bezüglich Qualität und Effizienz und einer longitudinalen Betrachtung von Krankheitsverläufen, die gerade in der Onkologie entscheidende Hinweise beinhalten können, nicht einmal ansatzweise realisierbar sein.

Der Anspruch an die ePA ist gewaltig. Unter Beibehaltung der in unserem Gesundheitssystem fest verankerten freien Arzt-, Krankenhaus- und Versicherungswahl werden die Daten unabhängig vom Entstehungsort in einer patientenspezifischen Akte gespeichert. Die dafür notwendige Speicherinfrastruktur wird den Versicherten von deren gesetzlichen Krankenkassen zur Verfügung gestellt. Da die medizinischen Daten dem Patienten gehören, entscheidet allein der Versicherte, ob es (s)eine persönliche Akte gibt und wer Zugriff auf die Daten hat. Natürlich wird die ePA von Ärzten angelegt und gefüllt, somit müssen sich 
auch die Leistungserbringer beteiligen. Gelingt das Projekt, braucht die deutsche medizinische Versorgung auch in $\mathrm{Zu}$ kunft keinen Vergleich mit anderen Ländern zu scheuen. Damit das „Jahrhundertprojekt" ePA erfolgreich wird, muss der Nutzen für alle Beteiligten jedoch von Anfang an klar erkennbar sein. Nur dann folgt die notwendige Akzeptanz. Ohne Akzeptanz wird die ePA genau da landen, wo der digitale Personalausweis seit seiner Einführung ruht: auf dem Friedhof gut gemeinter, aber schlecht gemachter digitaler Technologieprojekte.

\section{Wo liegt der Nutzen der ePA?}

Für den Versicherten besteht erstmals die Möglichkeit, alle relevanten medizinischen Daten zu speichern und damit sicherzustellen, dass nichts verloren geht. Da die Daten der Haus- und Fachärzte, Krankenhäuser und Rehabilitationseinrichtungen sowie der Versicherten selbst in die Akte einfließen, sind erstmals longitudinale Betrachtungen möglich - über die bestehenden Sektorengrenzen hinweg. Dies allein ist schon ein riesiger Fortschritt, der die Qualität der medizinischen Versorgung erheblich verbessern wird.

Darüber hinaus eröffnet sich mit der ePA eine Vielzahl weiterer Funktionalitäten, wie Arzneimitteltherapiesicherheit oder sichere digitale Arzt-Patient-Kommunikation, wobei Medikamente elektronisch verschrieben oder Arbeitsunfähigkeitsbescheinigungen online ausgestellt werden können.

Auch für die Behandelnden birgt die ePA zahlreiche Vorteile. Durch den Wegfall von Doppel- und Routinedokumentationen werden Ärzte und Pflegende entlastet. In die Arztpraxis kommen nur noch diejenigen, die wirklich einen Arzt sehen müssen. Die vielen Menschen, die einmal im Quartal ihr Rezept abholen oder Routinedaten liefern, könnten online versorgt werden.

Mittel- und langfristig werden die elektronisch verfügbaren Daten jedoch auch v. a. dazu beitragen, Diagnostik und Therapie weiter zu verbessern. Bezüglich des enormen Potenzials kratzen wir mit unserer Vorstellungskraft im Augenblick noch weitgehend an der Oberfläche.

Forum 2020 -35:300-303 https://doi.org/10.1007/s12312-020-00804-0

(c) Springer Medizin Verlag GmbH, ein Teil von Springer Nature 2020

\section{J. F. Debatin . C. Dirks \\ Die digitale Transformation der Medizin}

\section{Zusammenfassung}

Die Medizin wird sich in den kommenden Jahren weiter personalisieren. Entscheidende Grundlage für eine individualisierte Diagnostik und Therapie ist der Zugriff auf alle relevanten Patientendaten in Form einer elektronischen Patientenakte (ePA), gepaart mit einem digitalen Instrumentarium, um die richtigen therapeutischen Schlüsse aus der vorhandenen Datenflut zu ziehen. Entsprechend wird die Digitalisierung der Gesundheitsversorgung von drei zentralen Entwicklungen angetrieben: 1) besseres Verständnis der biologischen Lebensgrundlagen durch den breiteren Einsatz von DNA-Sequenzierungen, 2) technologische Quantensprünge bei Computing und Datenspeicherung, gepaart mit dem ubiquitären Zugang zu Daten über
Smartphones sowie 3) deutlich gestiegenes Souveränitätsbedürfnis der Patienten auf der Basis zunehmender Transparenz und digitaler Kommunikation. Die Digitalisierung wird somit Mittel zum Zweck einer verbesserten Gesundheitsversorgung. Dabei ist die Erhaltung der menschlichen Komponente entscheidend. Erst eine "humane Digitalisierung", in der der Nutzen für Patienten sowie für Behandelnde und Pflegende im Mittelpunkt steht, wird die vorhandenen Potenziale auch heben.

Schlüsselwörter

Personalisierte Medizin - Elektronische Patientenakte - Datenverarbeitung · Digitalisierung . Versorgungsqualität

\section{The digital transformation of medicine}

Abstract

Medicine will continue to become more personalized over the coming years. Beyond the availability of a central data repository in the form of an electronic patient record (ePA), the reaching of optimal diagnostic and therapeutic conclusions requires digital tools capable of handling the enormous flood of data. Accordingly, digitization of medicine is driven by three central developments: 1) better understanding of the biological basis of life, based on the wider use of DNA sequencing, 2) technological quantum leaps in computing and data storage coupled with ubiquitous access to data via smartphones; and 3) a considerable increase in patients' desire for sovereignty based on increasing transparency and digital communication. Thus, the digitization of medicine is evolving into a means for better health care. Despite all technological advances, it is important to continue focusing on the human component-only a "humane digitization" strategy, which places benefits for patients as well as those providing treatment and care at its core, will realize their full potential.

\section{Keywords}

Personalized medicine $\cdot$ Electronic medical record - Data processing · Digitization · Health care quality

\section{Weichenstellung muss jetzt erfolgen}

Damit die ePA tatsächlich ihren ganzen Nutzen entfalten kann, sind in den kommenden Wochen und Monaten noch einige Hürden zu nehmen. Hier geht es nicht zuletzt auch um technische Eckpfeiler wie die Interoperabilität der unterschiedlichen Systeme, sodass Informationen in ubiquitär verständlicher Weise zusammengeführt werden können.

Des Weiteren geht es um die inhaltliche Ausgestaltung, die einen direkten
Einfluss auf ihre Akzeptanz haben wird. Beispielsweise ist aus Sicht der Behandelnden ein arztgeführter Patientenpass zwingend. Dabei handelt es sich um eine Art Deckblatt oder 1-Blatt-Dossier, das neben den relevanten Diagnosen die aktuelle Medikation sowie Allergien auf einen Blick sichtbar macht. Entlassungsbriefe aus Krankenhäusern und Rehabilitationseinrichtungen sollten ebenso Teil der ePA sein wie die Dokumentation der stationären und ambulanten Pflege. Darüber hinaus wäre ein Aggregationstool für Laborwerte, mit dem alle Werte im 


\begin{tabular}{|l}
\hline \\
\hline \\
\hline Infobox Was Informationstech- \\
nologie leisten muss \\
\hline Personalisierte Medizin ist eine Querschnitts- \\
disziplin: Der praktische Arzt, der Onkologe, \\
der Pathologe müssen miteinander kom- \\
munizieren, dazu das Labor, die Kassen und \\
schließlich der Patient selbst. Das funktioniert \\
nicht ohne Informationstechnologie (IT). \\
Integration \\
Bei Genomanalysen entstehen große \\
Datenmengen, die nur mit IT ausgewertet \\
werden können. Die molekularen \\
Patienteninformationen müssen überprüft \\
und abgeglichen werden. Für den \\
Abgleich dienen wissenschaftliche \\
Erkenntnisse, klinische Befunde und bekannte \\
Wirkmechanismen von Medikamenten \\
- also konkretes Wissen aus 23 Mio. \\
medizinisch-wissenschaftlichen Artikeln. \\
Interpretation \\
Ärzte brauchen ein System, das die \\
molekularen Daten ihres Patienten aufnimmt \\
und automatisch prozessiert, also aus ihnen \\
das individuelle Krankheitsbild aufbaut. \\
Im Ergebnis heißt es dann nicht etwa „Der \\
Patient hat Darmkrebs“, sondern „Er hat einen \\
Krebs mit einem speziellen XY-Profil.“ Im \\
Idealfall erstellt das System darauf basierend \\
eine optimierte Behandlungsempfehlung. \\
Visualisierung und Pflege \\
Die Daten müssen für den Arzt so \\
aufgearbeitet, zusammengestellt und \\
präsentiert werden, dass er sie versteht und \\
intuitiv anwenden kann. \\
\hline
\end{tabular}

Zeitstrahl dargestellt werden, ausgesprochen wünschenswert.

Die digitale Transformation der Medizin birgt ein riesiges Potenzial. Für all die Punkte, die eine bessere Medizin generieren und die Behandlungsqualität verbessern werden, ist die Digitalisierung jedoch nur Mittel zum Zweck. Entscheidend bei all dem ist die menschliche Komponente - erst eine „humane Digitalisierung ", in der der Nutzen für Patienten sowie für Behandelnde und Pflegende im Mittelpunkt steht, wird das Potenzial auch heben.

\section{Korrespondenzadresse}

\section{Claudia Dirks}

hih - health innovation hub, Bundesministerium für Gesundheit Torstraße 223, 10115 Berlin, Deutschland claudia.dirks@hih-2025.de
Prof. Dr. med. Jörg F. Debatin leitet seit März 2019 als Chairman den health innovation hub des Bundesministeriums für Gesundheit (BMG). Als Ärztlicher Direktor und Vorstandsvorsitzender des Universitätsklinikums Hamburg-Eppendorf trug er bis 2011 durch konsequente Digitalisierung zu dessen erfolgreicher, papierfreier Erneuerung bei. Danach folgten Stationen als CEO der amedes AG und Vice-President bei GE Healthcare.

\section{Einhaltung ethischer Richtlinien}

Interessenkonflikt. J.F. Debatin und C. Dirks geben an, dass kein Interessenkonflikt besteht.

Für diesen Beitrag wurden von den Autoren keine Studien an Menschen oder Tieren durchgeführt. Für die aufgeführten Studien gelten die jeweils dort angegebenen ethischen Richtlinien.

\section{Literatur}

1. Nida-Rümelin J, Weidenfeld N (2018) Digitaler Humanismus. Eine Ethik für das Zeitaler der Künstlichen Intelligenz. Piper

\section{Aktuelle Informationen}

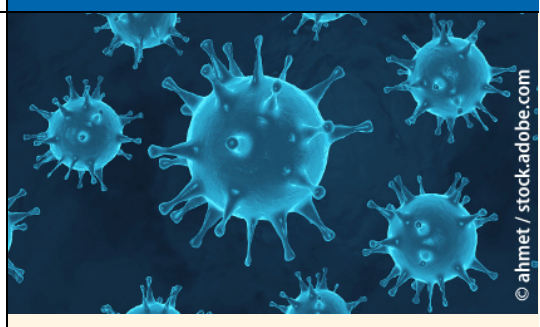

\section{Aktuelle Entwicklung zu COVID-19 bei Springer Nature und Springer Medizin}

Springer Nature und Springer Medizin unterstützen die globale Reaktion auf die COVID-19-Pandemie, indem ein schneller und direkter $\mathrm{Zu}$ gang zu den neuesten verfügbaren Forschungsergebnissen und Daten ermöglicht wird.

Auf der Homepage SpringerMedizin.de finden Sie ein immer aktuelles Dossier mit Beiträgen, Forschungsarbeiten und Ergebnissen zu SARS-CoV-2 sowie relevanten Links.

Darin z.B. auch die kürzlich publizierte

Empfehlung von DIVI, DGIIN, DGAI und DGP zur Intensivtherapie von Patienten mit COVID-19.

Springer Nature arbeitet mit globalen Organisationen zusammen, und verlinkt über SpringerNature.com/de auf eine eigene Landingpage mit einer Vielzahl an Information sowie freiem Zugriff auf die COVID-19-Contentplattformen von Nature Research, BioMed Central (BMC) und Springer.

Das Dossier zu Coronavirus / Covid-19 von Springer Medizin finden Sie hier: www.springermedizin.de/covid-19

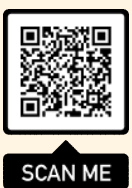

